\title{
New CAC Algorithm using Adaptive Modulation Control
}

\author{
Sung-Kee Noh ${ }^{1}$, Young-Ha Hwang ${ }^{1}$, Byung-Ho $\mathrm{Ye}^{1}$, and Sang-Ha Kim ${ }^{2}$ \\ 1 Electronics and Telecommunications Research Institutes, Daejon, Korea, \\ \{sknoh, hyh, bhye\}oetri.re.kr \\ 2 ChungNam National University, Daejon, Korea, \\ shkim@cnu.ac.kr
}

\begin{abstract}
Adaptive modulation control (AMC) has been proposed as the next generation modulation method for increasing network performance in cellular networks. Adaptive modulation is a powerful technique to improve the spectral efficiency in wireless transmission over fading channels. Similarly, it is possible to apply the adaptive modulation technique to Call Admission Control (CAC) scheme in order to enhance network performance and satisfy Quality of Service (QoS) requirements. In this paper, we investigate a novel call admission control (CAC) mechanism in the cellular networks using AMC. First, we build the system model in which takes into account to both CAC and AMC. Second, we verify that the CAC model can offer better performance by using adaptive modulation technique. Finally we prove our claim by numerical analysis.
\end{abstract}

\section{Introduction}

In multimedia wireless communication networks, the demand for high data rates and quality of service is growing at a rapid speed. In order to meet these needs, it is necessary to use more intelligent functions such as resource management, transmission technology, and network management. Thus, the proposed technology is Call Admission Control (CAC) with adaptive channel reservation (ACR) and adaptive modulation control(AMC).

The modulation method in communication systems have been introduced to meet finite frequency spectrum and efficient power control. The modem composed by the modulation method has power margin to satisfy time-based variable channel requests. The technique to transmit data efficiently using this power margin is called adaptive modulation, which is to enhance QoS and system throughput by changing the modulation method adaptively according to the instantaneous propagation conditions, interference scenarios, and traffic or data rate requirements. That is, adaptive modulation techniques do not require additional resources such as power and bandwidth according to variable traffic environment. Many previous adaptive modulation schemes have been suggested to maximize the data rate and match transmission parameters to time-varying channel conditions related to variable-power and variable-rate [1]-[6].

Please use the following format when citing this chapter:

Noh, S.-K., IIwang, Y.-II., Ye, B.-II. and Kim, S.-II., 2007, in IIIP International Federation for Information Processing, Volume 229, Network Control and Engineering for QoS, Security, and Mobility, IV, ed. Gaïti, D., (Boston: Springer), pp. $173-186$. 
A CAC scheme deals with the problem of whether or not a call is accepted into the network while taking QoS constraints into account in a given cell capacity. Thus, the objective of CAC scheme is to minimize handoff-call-dropping probability (CDP) and new-call-blocking probability (CBP), and to maximize the utilization of the assigned cell capacity at the same time. Generally, reserved guard channel schemes in which a certain number of channels from overall cell capacity have to be reserved solely for the use of handoff and new calls for different services have proposed to minimize CDP and CBP [7].

This paper evaluates the performance of new CAC algorithm using the advantages of practical AMC environments. Without AMC schemes, the system allocates new different frequency channel to user when the quality of the communications channel degrades. If the new channel belongs to the same base station, this is referred to as an intracell handoff. If we use AMC techniques, the system can reuse the same frequency channel under worse channel degradation. This leads to decreasing intracell handoff as well as increasing the utilization of system resources. In this paper, we analyze the joint effects of $\mathrm{CAC}$ and $\mathrm{AMC}$ techniques considering the user distance and signal strength.

The rest of this paper is organized as follows. We introduce the traffic model and user mobility characteristics in Section 2. We present an analytical procedure to solve the two-state markov chain in Section 3, and suggest the CAC algorithm and adaptive channel reservations policy in Section 4. Numerical results are discussed in Section5, and finally draw concluding remarks in Section 5 .

\section{System Model}

\subsection{Traffic Model}

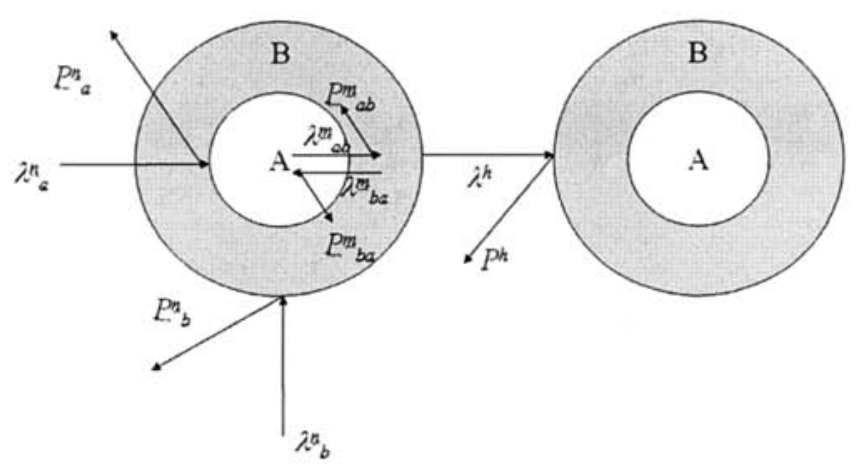

Fig. 1. System Model for a cell. 
The base station architecture is illustrated in Fig. 1. The idea behind adaptive modulation is to select a modulation mode according to the instantaneous radio channel quality [8]. We assume that different modulation is expressed by virtual region in a cell. Namely, calls in region $\mathrm{A}$ use modulation $\mathrm{A}$. We assume that each cell is equipped with same $C$ channels. For each cell $k$, there are two types of calls sharing these channels: the calls in region $A$ using modulation $A$ and the calls in region $B$ using modulation $B$. Region $B$ call requires $m$ bandwidths. Region $A$ call requires one basic bandwidth. It is assumed that the new calls in $A, B$ are arrived according to a Poisson process with mean arrival rate $\lambda_{a}^{n}=\lambda^{n} p_{a}$ and $\lambda_{b}^{n}=\lambda^{n} p_{b}$, respectively and that service time(call holding time) is exponentially distributed with mean service time of $1 / \mu_{a}^{n}$ and $1 / \mu_{b}^{n}$. Where, the probability $p_{a}\left(p_{b}\right)$ that a given call in a cell occurs in region $A(B)$ is

$$
p_{a}=\frac{\text { region } A}{\text { region } A+\text { region } B} \quad \text { and } \quad p_{b}=1-p_{a}
$$

When modulation mode switching is occurred, new channel should be allocated for the moving call in order to satisfy the Quality of Service (QoS) requirements. The intracell moving calls between $A$ and $B$ are generated with arrival rate $\lambda_{a b}^{m}$ and $\lambda_{b a}^{m}$, respectively. In next section we will describe the algorithms to generate $\lambda_{a b}^{m}$ and $\lambda_{b a}^{m}$. The intercell handoff occurs only in region $B$ with arrival rate $\lambda^{h}$ and that service time is exponentially distributed with mean service time of $1 / \mu^{h}$. Furthermore, the time that calls stay in the cell before moving into other cells also follows an exponentially distribution with mean $1 / h_{a}$ and $1 / h_{b}$. We also describes that calls in region $A$ and $B$ calls are summed with arrival rates $\lambda_{a}\left(\lambda_{a}^{n}+\lambda_{b a}^{m}\right)$ and $\lambda_{b}\left(\lambda_{b}^{n}+\lambda_{a b}^{m}+\lambda^{h}\right)$, respectively. Moreover channel occupancy times for region $A$ and $B$ calls are summed with means $1 / \mu_{a}\left(1 /\left(\mu_{a}^{n}+\mu_{b a}^{m}+h_{a}\right)\right)$ and $1 / \mu_{b}\left(1 /\left(\mu_{b}^{n}+\mu_{a b}^{m}+\mu^{h}+h_{b}\right)\right)$, respectively. Let $C$ be the total number of channels and $G_{a}$ and $G_{b}$ be the dedicated channels for region $A$ and region $B$ traffic, respectively. Then, the system can be modelled as a two dimensional Markov process shown in Fig. 2, characterized by $\{i, j\}$, where $i$ and $j$ are the numbers of calls in region $A$ and $B$, respectively and the state space is represented by the set set $\left\{s(i, j) \mid 0 \leq i \leq G_{a}, 0 \leq j \leq\left\lfloor\left(C-G_{b}\right) / m\right\rfloor\right.$ and $\left.G_{b} \leq i \leq C-G_{a}, 0 \leq j \leq\lfloor(C-i) / m\rfloor\right\}$. $\lfloor x\rfloor$ denotes the greatest integer smaller than or equal to $x$.

\subsection{Handoff rate for Distance and Signal Strength}

Generally, it is assumed that the new calls are arrived according to a Poisson process. However, the assumption of handoff or adaptive modulation change events being Poisson Process may no longer be practical. Some field studies $[9],[10],[11]$ showed that the channel occupancy times having an influence on handoff or adaptive modulation change events are not exponentially distributed for cellular systems. Thus, handoff call traffic can be expressed as a arrival rate $\lambda^{h}=\lambda E[H]$, where $E[H]$ is the average number of handoff calls induced [12]. Handoff or adaptive modulation change events initiation may be based on the 

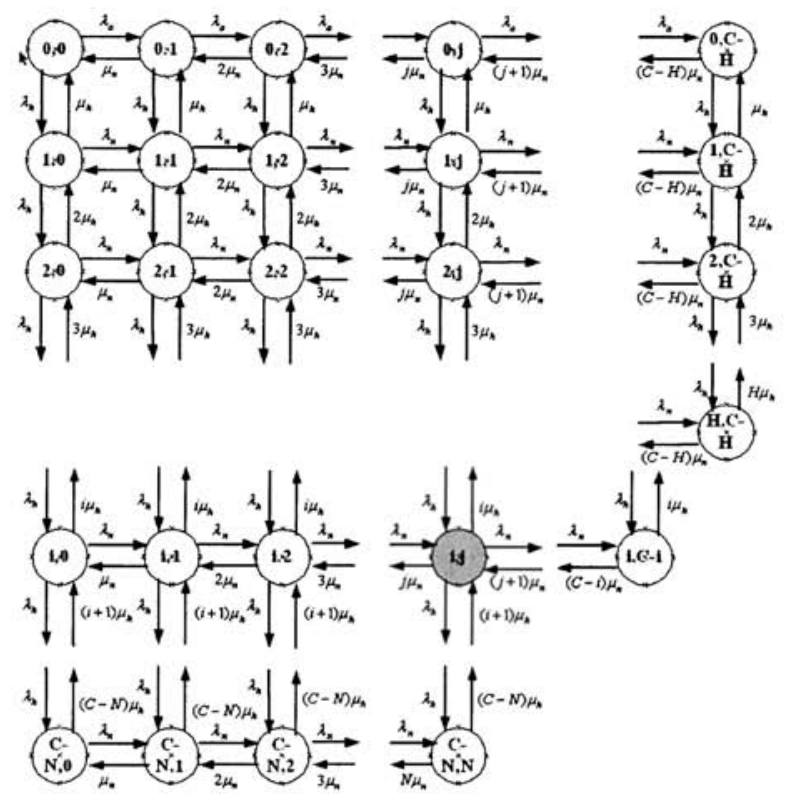

Fig. 2. The state diagram of call occupancy in region $A$ and $B$.

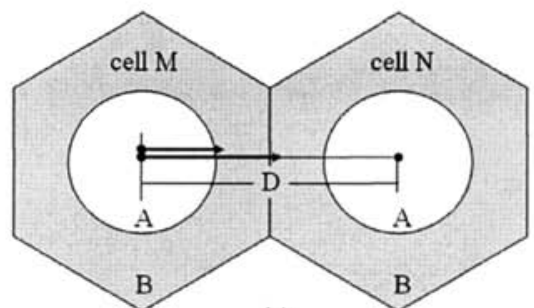

(a)

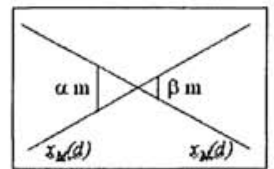

(b)

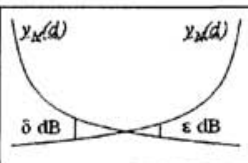

(c)

Fig. 3. Network model. (a) cellular layout (b) values of distance and (c) values of signal strength. 
distance between the mobile station and surrounding base stations, and the signal strengths the mobile station receives from base stations.

Itoh [13]proposed handoff algorithm that take account into distance and signal strength at the same time. Using Itoh [13]'s algorithm, we consider a network of two base station $M$ and $N$ separated by distance $D$, and a mobile station that is moving from $A$ to $B$ in a straight line with constant velocity (Fig. 3(a)). The algorithm considered performs a moving from area $A$ to $B$ within cell $M$ if both of the following conditions are met, as illustrated in Fig. 3(b).

1. if the measured signal strength from the adjacent area or base station exceeds that of the serving area or base station by a hysteresis level $\delta$ or $\epsilon(\mathrm{dB})$, respectively;

2. if the measured distance from the serving adjacent area or base station exceeds that of the adjacent area or station by a threshold distance $\alpha$ or $\beta(\mathrm{m})$, respectively.

At a distance $d \mathrm{~m}$ from base station $M$, the estimated distance from base stations $M$ and $N$ is given by

$$
x_{M}(d)=d+n_{M}
$$

and

$$
x_{N}(d)=(D-d)+n_{N}
$$

where $n_{M}$ and $n_{N}$ represent distance measurement error and are modeled as zero-mean independent white Gaussian processes with variance $\sigma_{N}^{2}$. The relative distance is defined as

$$
x(d)=x_{M}(d)-x_{N}(d)=2 d-D+n .
$$

where $n=n_{M}-n_{N}$ is zero-mean white Gaussian process with variance $\sigma^{2}=2 \sigma_{n}^{2}$.

The signal levels received from base station $M$ and $N$, are given by

$$
y_{M}(d)=-K \log (d)+\mu(d)
$$

and

$$
y_{N}(d)=-K \log (D-d)+\nu(d)
$$

Following the procedure used in [14], let $P_{h}(k)$ denote the probability that there is a handoff at interval $k . P_{M N}(k)$ denotes the probability of handoff from $M$ to $N$, and vice versa for $P_{N M}(k)$. Then, if $P_{M}(k)$ and $P_{N}(k)$ denote the probability that the mobile is assigned to base station $M$ or $N$ at interval $k$, the following recursive relations hold:

$$
\begin{array}{r}
P_{h}(k)=P_{M}(k-1) P_{M N}(k)+P_{N}(k-1) P_{N M}(k) \\
P_{M}(k)=P_{M}(k-1)\left(1-P_{M N}(k)\right)+P_{N}(k-1) P_{N M}(k) \\
P_{N}(k)=P_{M}(k-1) P_{M N}(k)+P_{N}(k-1)\left(1-P_{N M}(k)\right) .
\end{array}
$$


It is clear that once we find a way to compute $P_{M N}(k)$ and $P_{N M}(k)$, the problem is solved.

$$
\begin{aligned}
& P_{M N}(k)=P\{N(k) \mid M(k-1)\} \\
& \quad=P\left\{y_{k}<-\varepsilon, x_{k}>\beta \mid M(k-1)\right\} \\
& \quad=P\left\{y_{k}<-\varepsilon \mid M(k-1)\right\} P\left\{x_{k}>\beta \mid M(k-1)\right\} .
\end{aligned}
$$

$P_{A B}(k), P_{B A}(k)$ can be calculated in a similar fashion, i.e.,

$$
\begin{array}{r}
P_{m}(k)=P_{A}(k-1) P_{A B}(k)+P_{B}(k-1) P_{B A}(k) \\
P_{A}(k)=P_{A}(k-1)\left(1-P_{A B}(k)\right)+P_{B}(k-1) P_{B A}(k) \\
P_{B}(k)=P_{A}(k-1) P_{A B}(k)+P_{B}(k-1)\left(1-P_{B A}(k)\right) .
\end{array}
$$

It is clear that once we find a way to compute $P_{A B}(k)$ and $P_{B A}(k)$, the problem is solved.

$$
\begin{aligned}
& P_{A B}(k)=P\{B(k) \mid A(k-1)\} \\
& \quad=P\left\{y_{k}<-\varepsilon, x_{k}>\beta \mid A(k-1)\right\} \\
& =P\left\{y_{k}<-\varepsilon \mid A(k-1)\right\} P\left\{x_{k}>\beta \mid A(k-1)\right\} .
\end{aligned}
$$

Then, the handoff call attempt rate per cell is expressed as

$$
\begin{aligned}
& \lambda_{a b}^{m}=\lambda_{a}^{n} \cdot \sum k P_{A B}(k) \\
& \lambda_{b a}^{m}=\lambda_{b}^{n} \cdot \sum k P_{B A}(k) .
\end{aligned}
$$

\section{Analysis with MMPP}

The steady-state probability vector $p$ is then partitioned as $p=\left(p_{0}, p_{l}, \ldots\right)$. The vector $p$ is the solution of equations

$$
p Q=0, \quad p e=1
$$

Where $\boldsymbol{e}$ and $\boldsymbol{O}$ are vectors of all ones and zeros, respectively, and $\boldsymbol{Q}$ is the transi-tion rate matrix of the Markov process which will be obtained for each allocation strategy. we can obtain the transition rate matrix $Q$ of the Markov process

$$
Q=\left[\begin{array}{cccccc}
A_{0} & D & & & \\
B_{1} & A_{1} & D & & \\
& B_{2} & A_{2} & D & \\
& & B_{3} & A_{3} & D \\
& & & \cdot & \cdot & \cdot \\
& & & & & \cdot
\end{array}\right]
$$


Let $p_{i,-1}=0$ for $0 \leq i \leq C-G_{b}$ and $p_{-1, j}=0$ for $0 \leq j \leq\left\lfloor\left(C-G_{a}\right) / m\right\rfloor$. We show some balance equations as follows.

$$
\begin{gathered}
0 \leq i \leq G_{a}, 0 \leq j \leq\left\lfloor\left(C-G_{a}\right) / m\right\rfloor: \\
\left(\lambda_{a}+i \mu_{a}+\lambda_{b}+j \mu_{b}\right) p_{i j}=\lambda_{a} p_{i-1, j}+(i+1) \mu_{a} p_{i+1, j}+ \\
\lambda_{b} p_{i, j-1}+(j+1) \mu_{b} p_{i, j+1} \\
0 \leq i \leq G_{a}-1,\left\lfloor\left(C-G_{a}\right) / m\right\rfloor \leq j \leq\left\lfloor\left(C-G_{a}-1\right) / m\right\rfloor: \\
\left(\lambda_{a}+i \mu_{a}+\lambda_{b}+\left\lfloor\left(C-G_{a}\right) / m\right\rfloor \mu_{b}\right) p_{i j}=\lambda_{a} p_{i-1, j}+ \\
(i+1) \mu_{a} p_{i+1, j}+\lambda_{b} p_{i, j-1}+\lfloor(C-G n) / m\rfloor \mu_{b} p_{i, j+1} \\
0 \leq i \leq G_{a}, j=\left\lfloor\left(C-G_{a}\right) / m\right\rfloor: \\
\left(\lambda_{a}+i \mu_{a}+\left\lfloor\left(C-G_{a}\right) / m\right\rfloor \mu_{b}\right) p_{i j}=\lambda_{a} p_{i-1, j}+(i+1) \\
\mu_{a} p_{i+1, j}+\lambda_{b} p_{i, j-1} .
\end{gathered}
$$

Equations (15) maybe written concisely in matrix form. To do this define a set of $\left(C-G_{a}\right)$ elements row vector $\boldsymbol{p}_{i}$.

$$
p_{i} \equiv\left[p_{i 0}, p_{i 1}, p_{i 2}, \ldots\right] \text {. }
$$

From above equations (16), we can define submatrices for $i, j=0,1, C$ $G_{b}, 0 \leq l \leq\left\lfloor\left(C-G_{a}\right) / m\right\rfloor$ by

$$
\begin{gathered}
A_{l}(i, j)= \begin{cases}\lambda_{a} & \text { if } i=j-1 \text { and }\left(0 \leq i \leq G_{a} \mid i<C-l \cdot m\right) \\
j \mu_{a} & \text { if } i=j+1 \text { and } i \leq C-l \cdot m \\
a_{i}(j) & \text { if } i=j \\
0 & \text { otherwise. }\end{cases} \\
D(j, k)= \begin{cases}\lambda_{w} & \text { if } i=j \text { and } i \leq C-l \cdot m \\
0 & \text { otherwise. }\end{cases} \\
B_{l}(i j)= \begin{cases}\text { nin }\left(l,\left\lfloor\left(C-G_{a}\right) / m\right\rfloor,\lfloor(C-i) / m]\right) \text { aif } i=j \text { and } i \leq C-l \cdot m \\
\varrho & \text { otherwise. }\end{cases}
\end{gathered}
$$

Where $a_{i}(j)$ is the value that makes the sum of the row element s of $Q$ equal to zero.

\section{The CAC Algorithm}

Channels are divided by three sub-channels. Designed $G_{b}$ and $G_{a}$ channels are dedicated for region $B$ traffic and region $A$ traffic, respectively. The shared channels can be used by either type of traffic. When a new user arrives in a cell, the proposed CAC algorithm decides acceptance or rejection based on each call's current resource occupancy, reservation partition, and dynamic guide channels. A new region $B$ call is admitted if the number of existing region $B$ calls is less than the number of guard channels $G_{b}$ for region $B$ traffic. When the number of existing region $B$ calls is greater than or equal to the number of guard channels 
$G_{b}$ for region $A$ traffic, a new region $B$ call is accepted when the total existing used channels are less than a predefined threshold. Hand-off region $B$ calls are accepted as long as the channels are not full.

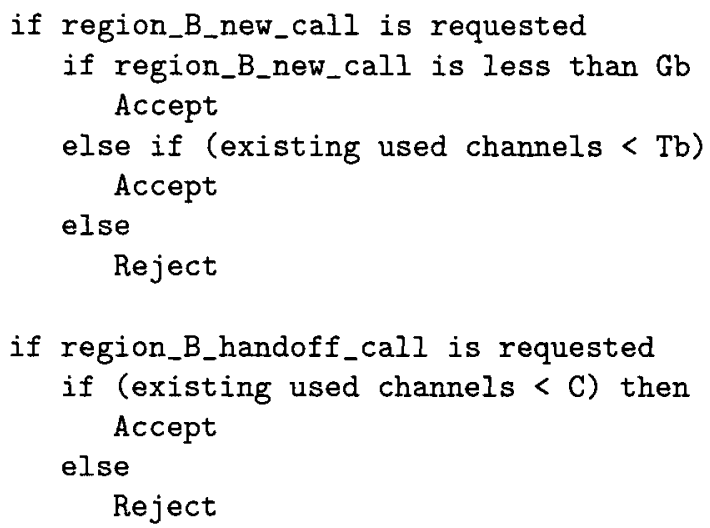

\section{The Adaptive Resource Management}

In our CAC algorithm described in chapter VI, we should compute $G_{a}$, and $G_{b}$. In this section, we describe how to decide reservation partition for region $A$ and region $B$ calls. These reservation partitions play a very important role in admission control and resource utilization, so that it is very critical problem to set these values properly. Each service call reservation partition is allocated with traffic behavior as well as fairness level. Each reservation partition has minimum channel pool to guarantee minimum resource to each service. Using minimum channel pool, it prevents all resources from being occupied by one service so that it can help to make Call Blocking Probability (CBP) ratio balance and achieve fairness for resource usage.

\subsection{Initialization}

Initial reservation partition for each class is proportionally allocated with the offered load per cell, which is defined as call generation rate ${ }^{*}$ required bandwidth units * average call staying time in a cell. So, the much resource is allocated to class with larger offered load than class with small offered load. The minimum channel pools for each class is set as the same as initial reservation partition. The computations are given by the following equations.

$$
\begin{aligned}
& G_{a}=C * \frac{\text { load_region_A } A}{\Sigma \text { load_region_A, } \text { load_region_B } B_{1}} * \alpha \\
& G_{b}=C * \frac{\text { load_region_B }}{\text { Eload_region_A, load_region_B }} * \alpha
\end{aligned}
$$

where, $\alpha$ has the value between 0 and 1 . 


\subsection{Adjustment}

Based on CBP_ratio, resource management algorithm works as follows. If CBP_ratio is greater than threshold, the reservation partition for underprivileged class is incremented up to sum of resource for expecting on-going calls over next term and required resources for CBP fairness. The former is calculated by call's departure rate and the latter is done by call's arrival rate. The required resource for CBP fairness are defined as calls.for_CBP_fairness * BU. The term calls_for_CBP_fairness means the relative number of calls, which should be admitted to make current unfair CBP fairness equal or similar. To compute these calls, at first current available resource is accomplished. This calculation is based on current occupied resource. Once calculating this resource, the remaining resources are partitioned depending on CBP fairness level and offered traffic load. That is, the CBP fairness level has a great important role in deciding call admission during next term. For example, if the CBP unfairness is serious, the bandwidths accommodating all expected calls are to be allocated. On the contrary, if the CBP unfairness is light, bandwidths for admitting several calls among all expected calls are to be demanded. As a result, it is very important to predict the number of calls for getting adequate resources for CBP fairness. Once completing estimation, system calculates how many calls should be admitted in order to lower CBP of unprivileged calls up to current CBP of privileged calls. The detail procedures for this computation are as follows. In order to make CBP fair, the calls_for_CBP_fairness are calculated by (1 - CBP of other service) * available_resource. This concept is based on biased coin method [15]. That is, the CBP restricts to the admitted calls over next terms. So, the CBP fairness can be gradually achievable. For better understand, we take an example. We assume that the CBP of service $i$ is $1 / 5$ and CBP of service $j$ is $1 / 4$. And, the available resources are 20 units where offered traffic load of each class is given as 1 and 2, respectively. According to our algorithm, the acceptance threshold of service $i$ and $j$ in next interval are set to $(1-(1 / 4))^{*} 20 * 1 / 3=5$ and ( $1-(1 / 5)) * 20 * 2 / 3=11$, respectively. So, during next interval, 5 units are reserved for service $i$ and 11 units are reserved for service $j$. The remaining 4 units are competed with two service class equally. Using this method, the CBP unfairness is gradually to be balanced.

If CBP_ratio > threshold and Then

a_r $=\mathrm{C}-\mathrm{Ri}$

$\mathrm{Gi}=\mathrm{R} i+\left(1-\mathrm{CBP}_{-} \mathrm{j}\right) * \mathrm{a}_{-} r * o_{-}$Ioad_i $_{-}$/o_load

$G j=R j+\left(1-C B P \_i\right) * a_{-} r * o_{-}$load $_{-} j / o_{-}$load

Else

Nothing is done 


\section{Numerical Results}

The objective of this numerical analysis is to verify the excellency of new CAC algorithm and adaptive channel allocation method in mobile network environment using adaptive modulation compared to using fixed modulation with respect to call dropping probability. The system parameters used in numerical analysis are show in Table 1.

Table 1. System Parameters.

\begin{tabular}{|c|c|c|}
\hline Name & Value & Explanation \\
\hline$C$ & 15 & cell capacity \\
$G_{a}$ & 2 & initial guard channel for $A$ \\
$G_{b}$ & 4 & initial guard channel for $B$ \\
$m$ & 2 & basic channel units for $B$ \\
$\mu_{a}$ & 1 & channel occupancy times for $A$ \\
$\mu_{b}$ & 1 & channel occupancy times for $B$ \\
$\lambda$ & $0.1-3$ & new call arrival rate \\
\hline
\end{tabular}

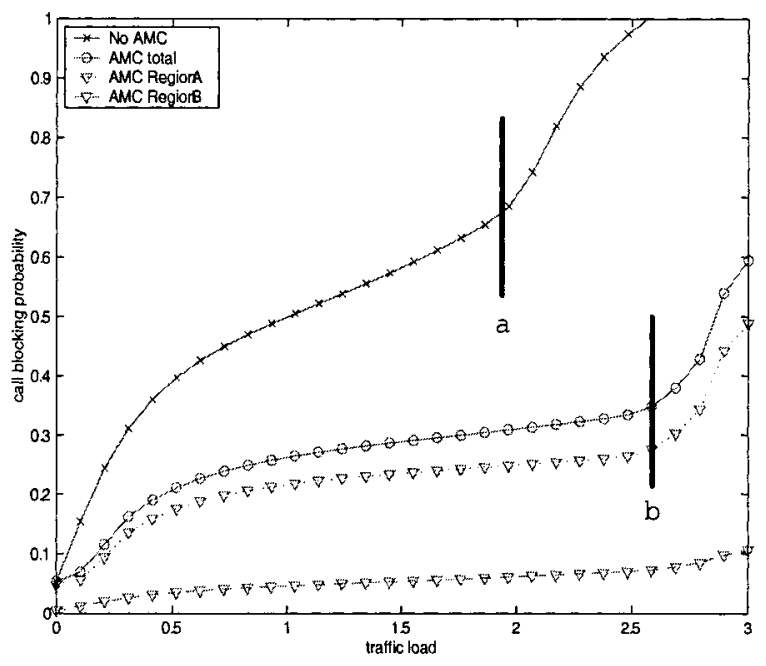

Fig. 4. Blocking probability (AMC vs No AMC.)

Fig. 4 shows the obvious distinction of call blocking probability between AMC and no AMC. Under fixed modulation (no AMC), it is clear that the new call 


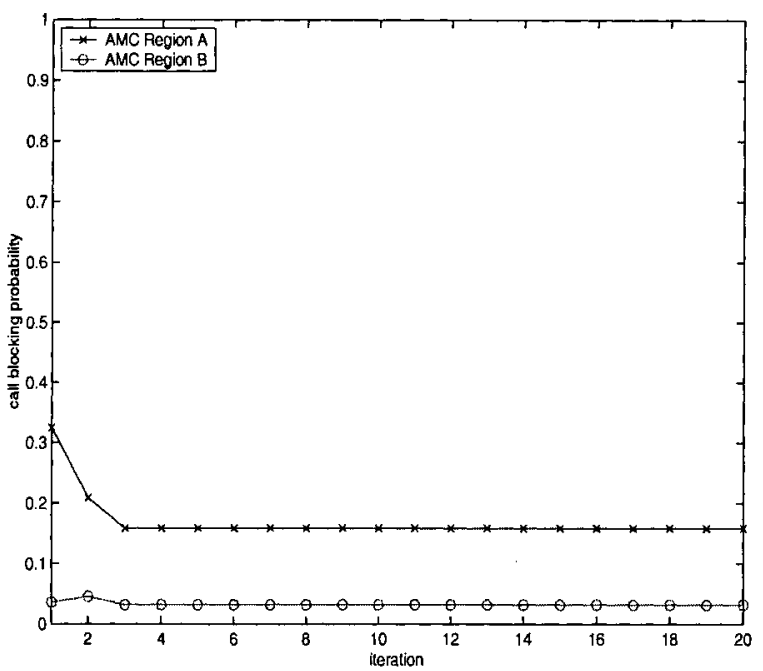

(a) $\rho=0.5$

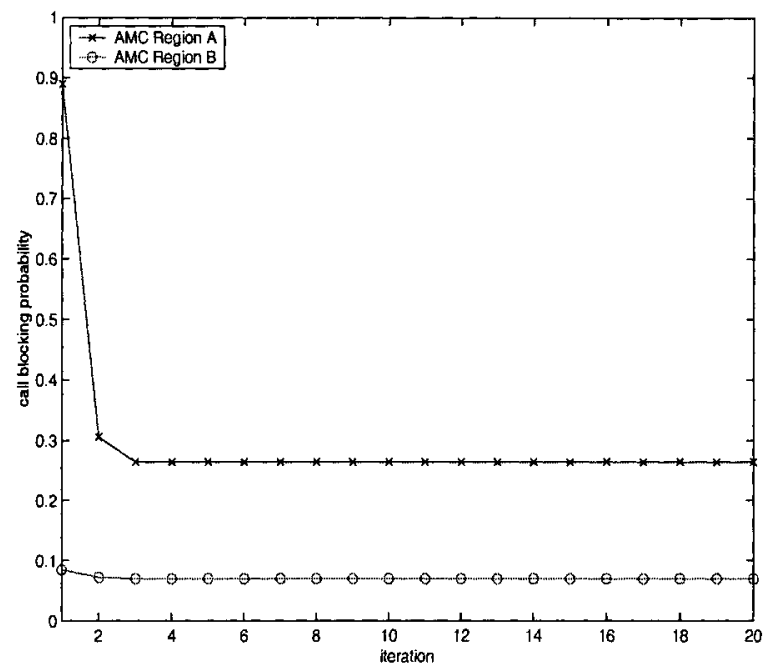

(b) $\rho=2.5$

Fig. 5. Blocking probability with $\rho=0.5$ and $\rho=2.5$.

blocking probability (CBP) increases drastically over $C B P_{t h}(0.3)$ in proportion to increase of traffic load $(\rho)$. On the contrary, under AMC environment, CBP is kept below $C B P_{t h}(0.3)$ until traffic load increase to the point of about 2.5. Fig. 5 shows that how our CAC scheme using adaptive resource management guarantees $C B P_{t h}$ after some iterations. The figure indicates that our scheme is 


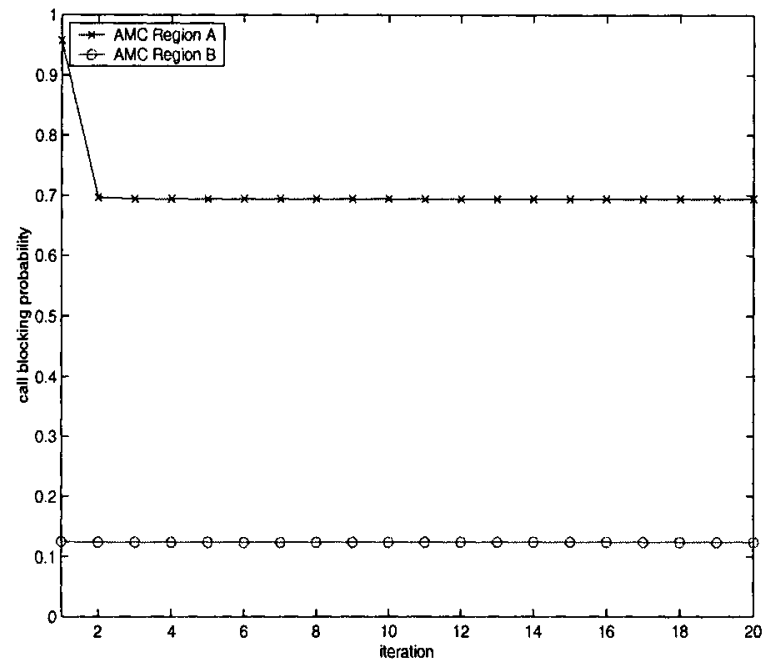

(a) No borrowing scheme

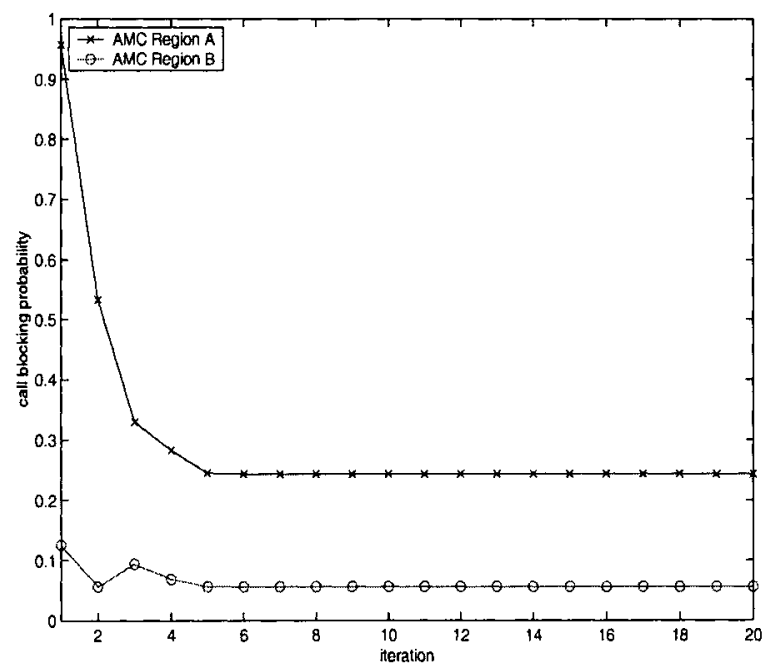

(b) With channel borrowing scheme

Fig. 6. Blocking probability using channel borrowing scheme with $\rho=5$

more efficient under high traffic load. For examples, at the first step of our CAC scheme, we also can not guarantee $C B P_{t h}=0.3$, even in case of traffic load ( $\rho=0.5$ ) as show in Fig. 5(a), but after short iteration, CBP converges under $C B P_{t h}$. Fig. 5(b) also reveals that CBP converges rapidly below $C B P_{t h}(0.3)$ with $\rho=2.5$ after 4 iterations of our algorithm, while no AMC shows that CBP 
is over 0.9 .

Fig. 6(a), however, shows that CBP of region A seems not to be converged within the $C B P_{t h}(0.3)$. It is because the traffic load is relatively higher than total channel capacity to handle input traffic. Namely, CBP for highly overloaded situation cannot be adjusted. Thus, we think that it is necessary to apply extra channel borrowing scheme from adjacent cells to solve this highly overload status. In Fig. $6(\mathrm{~b})$, we increase the traffic parameters until the CBP satisfies the $C B P_{t h}$. That is, we assume that cell capacity $(C)$ increases from 15 to 21 by borrowing extra cell channels from adjacent cells because the available channels are absolutely deficient to meet $C B P_{t h}$. Fig. 6(b) shows that CBP converges very well below $C B P_{t h}$ when channel borrowing scheme is used.

\section{Conclusion}

In this study we investigated the new CAC algorithm in wireless networks using adaptive modulation technique. The main concern of this work is to verify that the CAC model can offer better performance by using adaptive modulation technique that take into account user mobility property. The proposed method has been analyzed by conducting a two-dimensional Markov chain, and has been proven by using SOR solutions. Using numerical result, we demonstrated that our CAC scheme actually achieves resonable admitting probability regardless of traffic behavior. Adaptive modulation is a powerful technique to improve the spectral efficiency in wireless transmission over fading channels. Similarly, it is possible to apply the adaptive modulation technique to Call Admission Control (CAC) scheme in order to enhance network performance and satisfy Quality of Service (QoS) requirements.

\section{References}

1. J. S. Blogh, P. J. Cherriman, and L. Hanzo, "Dynamic Channel Allocation Techniques Using Adaptive Modulation and Adaptive Antennas," IEEE J. Select. Areas Commun., vol. 19, no. 2, pp. 312-321, Feb. 2001.

2. S. F. Falahati, A. Svensson, T. Ekman, and M. Sternad, "Adaptive Modulation Systems for Predicted Wireless Channels," IEEE Trans. Commun., vol. 52, no. 2, pp. 307-316, Feb. 2004.

3. B. Vucetic, "An adaptive coding scheme for time-varying channels," IEEE Trans. Commun., vol. 39, pp. 653.663, May 1991.

4. S. M. Alamouti and S. Kallel, "Adaptive trellis-coded multiple phaseshift keying for Rayleigh fading channels," IEEE Trans. Commun., vol. 42, pp. 2305.2314, June 1994.

5. M.-S. Alouini and A. J. Goldsmith, "Adaptive M-QAM modulation over Nakagami fading channels," in Proc. IEEE Global Communications Conf., Phoenix, AZ, Nov. 1997, pp. 218.223.

6. A. J. Goldsmith and S. Chua, "Variable-rate variable-power M-QAM for fading channels," IEEE Trans. Commun., vol. 45, pp. 1218.1230, Oct. 1997. 
7. J. Y. Lee, "Realistic cell-oriented adaptive ad-mission control for QoS support in wireless multimedia networks," IEEE Trans. Veh. Technol., vol. 52, no. 3, May 2003.

8. L. Hanzo, W. T. Webb, and T. Keller, Single- and Multi-Carrier Quadrature Amplitude Modulation. New York: Wiley/IEEE, 2000.

9. C. F. Barcelo and J. Jordan, "Channel Holding Time Distribution in Cellular Telephony," Proc. Ninth Int'l Conf. Wireless Comm. (Wireless 97), vol. 1, pp. 125-134, Alberta, Canada, 9-11 July 1997.

10. C. Jedrzycki and V.C.M. Leung, "Probability Distribution of Channel Holding Time in Cellular Telephony Systems," Proc. IEEE Vehicular Technology Conf. (VTC 96), pp. 247-251, 1996.

11. J. Jordan and F. Barcelo, "Statistical Modelling of Channel Occupancy in Trunked PAMR Systems," Proc. 15th Intl Teletraffic Conf. (ITC 15), V. Ramaswami and P.E. Wirth, eds., pp. 1,169-1,178.

12. Y. Fang and Y. B. Lin, "Channel Occupancy Times and Handoff Rate for Mobile Computing and PCS Networks," IEEE Trans. Computers, vol. 47, no. 6, pp. 679692, Jun. 1998.

13. K. I. Itoh, J. S. Shih, and T. Sato, "Performance of Handoff Algorithm Based on Distance and RSSI Measurements," IEEE Trans. Vehicular Technology, vol. 51, no. 6, pp. 1460-1468, Nov. 2002.

14. P. S. Kumar and J. Holtzman, "Aanlysis of Handoff Algorithms Using Both Bit Error Rate and Relative Signal Strength," in Proc. 3rd Annu. Int. Conf. Universal Personal Communications, 1994, pp. 1-5

15. L. J. Wei, "The Adaptive Biased Coin Design for Sequential Experiments," Journal of Annals of Statistics, Vol. 6, Jan. 1978, pp. $92-100$. 